\title{
Cylindrical periodic surface lattice as a metadielectric: Concept of a surface-field Cherenkov source of coherent radiation
}

\author{
I. V. Konoplev, ${ }^{*}$ A. J. MacLachlan, C. W. Robertson, A. W. Cross, and A. D. R. Phelps \\ SUPA, Department of Physics, University of Strathclyde, Glasgow G4 ONG, United Kingdom
}

(Received 8 April 2011; published 25 July 2011)

\begin{abstract}
A two-dimensional (2D), cylindrical, periodic surface lattice (PSL) forming a surface field cavity is considered. The lattice is created by introducing 2D periodic perturbations on the inner surface of a cylindrical waveguide. The PSL facilitates a resonant coupling of the surface and near cutoff volume fields, leading to the formation of a high- $Q$ cavity eigenmode. The cavity eigenmode is described and investigated using a modal approach, considering the model of a cylindrical waveguide partially loaded with a metadielectric. By using a PSL-based cavity, the concept of a high-power, 0.2-THz Cherenkov source is developed. It is shown that if the PSL satisfies certain defined conditions, single-mode operation is observed.
\end{abstract}

DOI: 10.1103/PhysRevA.84.013826

\section{INTRODUCTION}

Electromagnetic (EM) field excitation and evolution inside and on the surface of periodic structures facilitate interaction between active media and the fields and thus are important and challenging problems in plasma physics and electronics [1-10] as well as optics and photonics [11-17]. Extensive study of the electromagnetic wave propagation and control in periodic structures has already led to many technological breakthroughs and is a driving force behind many interesting concepts such as plasmonic devices [11-15], particle acceleration [3,4], and signal transformers [10,15-17]. Bridging the terahertz (THz) gap and realization of compact, high-power sources operating in the GHz-THz $[1,2,5-8]$ and $\mathrm{x}$-ray $[1,11]$, frequency ranges are also strongly linked to the ability to control the EM fields inside and on the surface of periodic lattices. In recent years, a large amount of research has been carried out using periodic lattices and deals with both propagating volume (bulk) waves and surface waves. For many conventional active devices, such as lasers, localized surface waves are rather inconvenient due to their strong localization at the surface accompanied by large thermal losses and weak coupling with bulk active media resulting from the rapid exponential decay inside the active media. However, it has been suggested recently that such fields can be used either in very small nano-oscillators [14] or in high-power $[5,6]$ active devices. The research has been further propelled [15-17] by the exponential development of nanofabrication and nanotechnology. The current stage is one of the rapidly growing areas of research, promising groundbreaking results in signal processing, communication [18], and spectroscopy [16]. The lattices (metamaterials) are normally based on fundamental cells (scatterers) (e.g., splitring resonators or nanoparticles covered with dielectrics) having dimensions much smaller than the operating wavelength $\lambda[19]$. Conventionally, both theory and experiments have been developed for these structures with overall transverse dimensions comparable with the operating wavelength, which allows synchronization of the radiation from individual scatterers. However, there are a number of challenges associated with the interaction region's small size, including manufacture of the

\footnotetext{
*ivan.Konoplev@strath.ac.uk
}

PACS number(s): 42.25.Fx, 42.60.Da, 78.67.Pt, 73.20.Mf

lattices and their output power limitations. A simple scaling up of the interaction region's dimensions (i.e., making the total surface area $S \gg \lambda^{2}$ or total volume $V \gg \lambda^{3}$ ) results in disruption of the coherent emission or scattering due to problems associated with synchronization of the individual scatterers that form the metamaterial. The synchronization of the individual scatterers is needed, for example, because of the spatial detuning (caused by finite tolerances of manufacturing) and temporal detuning (caused by nonuniform heating of the metamaterial) of the scatterers' eigenfrequencies and lack of "cross talk" and feedback between them. If the synchronization is not provided, the temporal and spatial coherence of such devices can be questionable. Overcoming these difficulties is especially important for applications where high-power, coherent radiation is required, for instance, in $\mathrm{THz}$ active devices for pollution monitoring (atmospheric dust clouds and space debris), security (active control and detection), chemistry, and bioscience. In this paper, we discuss one of the ways to synchronize the radiation from individual scatterers or radiators assembled into a large-area structure. The structure discussed is based on a cylindrical conducting waveguide having an area $S=2 \pi r_{0} L \gg \lambda^{2}$ and manufactured using electroforming techniques. The periodic two-dimensional (2D) perturbations on the inner surface of the waveguide have amplitudes much smaller than the operating wavelength $(\lambda)$ and form the 2D periodic surface lattice (2D PSL) of cylindrical topology. The photograph and numerical model of the 2D periodic structure are shown in Figs. 1(a) and 1(b). Each individual element of the lattice [Fig. 1(c)] has dimensions smaller but comparable with the operating wavelength $(\sim \lambda / 2)$, and each fundamental cell of the lattice is an individual scatterer that supports an individual localized surface field. The surface currents excited along the boundaries of the cells [Fig. 1(c)] allows "cross talk" between fundamental cells and coupling of the surface and near cutoff volume fields. These lead to synchronization of the scatterers' oscillations from different parts of the oversized structure. The structure studied [Figs. 1(a) and 1(b)] is low contrast (small amplitude perturbations) and oversized, allowing the "square wave" approximation (also known as the "chessboard" model) [5,7], to be used [Fig. 1(b)]. Further, in this paper, all numerical simulations are carried out using the "square wave" model. Figure 1(c) demonstrates the excitation of synchronized 
surface currents $I_{n}$ around a single cell $C_{n}$ formed by square wave perturbations. The contour plot [observed using the three-dimensional (3D) software package CST MICROWAVE STUDIO] demonstrates the current distributions on the surface of the individual cells $C_{n}$, while the arrows indicate the currents' flow directions. To study the eigenmodes of the structure (Fig. 1), the lattice, consisting of the discrete, distributed scatterers, is substituted with a cylindrical waveguide partially loaded with continuous "metadielectric" [19]. The parameters and the properties of the "metadielectric" such as its geometry and refractive index are discussed. We note that the introduction of the metadielectric allows us to consider the localized surface fields supported by the scatterers as eigenmodes of a partially loaded waveguide [20]. Such modes, being eigensolutions of the wave equation, can only be observed in a waveguide with resistive walls or partially loaded with dielectric. One of the features of such fields is an imaginary transverse wave number in free space, leading to localization of the surface modes inside the metal skin layer or the dielectric and decaying rapidly outside. Also, when increasing the field frequency toward the optical range and the metal plasma frequency, the surface fields become known as surface plasmons. In this work, the field structure is studied and a surface field (SF) Cherenkov source based on such a SF cavity will be designed. The results of numerical studies of the Cherenkov source driven by an oversized, mildly relativistic electron beam are presented and discussed.

The paper's structure is as follows. In Secs. II and III, the basic model and equations are described and shown. The model is analyzed and the results are discussed. In these sections, the results of the numerical studies which have been conducted using the 3D code MAGIC are presented and compared with analytical data. By substituting the periodic lattice with a metadielectric and combining this approach with direct 3D numerical modeling of the lattice, the understanding of the field evolution and formation of the cavity's eigenfield structure is developed. Section IV is dedicated to the concept of Cherenkov sources based on a 2D cylindrical lattice, and it is shown that such sources can produce spatially coherent high-power radiation in the high- $\mathrm{GHz}$ to $\mathrm{THz}$ frequency ranges. The basic principles of the SF Cherenkov source and its design are discussed. In the conclusion, we summarize the results obtained.

\section{CYLINDRICAL STRUCTURE BASED ON 2D LATTICE: ANALYTICAL AND NUMERICAL MODELS}

The 2D lattice of cylindrical topology [Fig. 1(a)] can be observed by machining small periodic perturbations $(\Delta r<<\lambda$, where $\Delta r$ is the amplitude of the perturbations) on the inner surface of the cylindrical waveguide: $r=r_{0}+$ $\Delta r \cos \left(\bar{k}_{z} z\right) \cos (\bar{m} \varphi)$. Here, $r_{0}$ is the mean radius of the unperturbed waveguide, $\bar{k}_{z}=2 \pi / d_{z}$ and $d_{z}$ is the lattice longitudinal period, and $\bar{m}$ is the lattice number of azimuthal variations. The structure made from copper [Fig. 1(a)] has a large diameter $2 r_{0}=79 \mathrm{~mm}\left(r_{0} \gg d_{z}=8 \mathrm{~mm}, r_{0} \gg\right.$ $\lambda, \lambda \sim 8 \mathrm{~mm}$ ) and $\bar{m}=28$. Let us note that $\bar{m}$ is smaller than $M=2 \pi r_{0} / d_{z} \sim 31$; that is, the cells forming the lattice are slightly asymmetric. Such a structure has already (a)

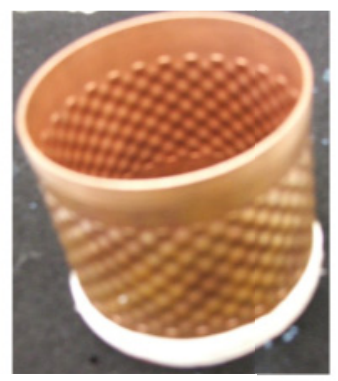

(b)

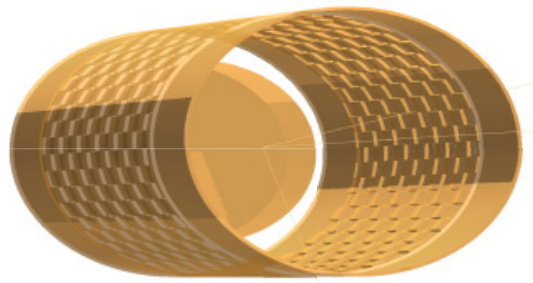

(c)

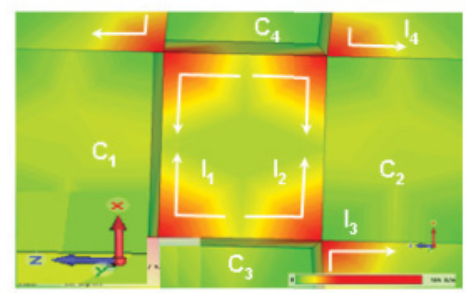

FIG. 1. (Color online) (a) Photograph of the 2D periodic lattice of length $L=48 \mathrm{~mm}$ machined on the inner surface of copper cylindrical waveguide of mean diameter $80 \mathrm{~mm}$. The lattice has longitudinal period $d_{\mathrm{z}}=8 \mathrm{~mm}$ and number of azimuthal variations $\bar{m}=28$ azimuthal. (b) The chessboard model [5,7], of the 2D cylindrical lattice studied via numerical simulations and illustrated using 3D code MAGIC. (c) The excitation of surface currents on the lattice fundamental cell boundaries observed using 3D code CST MICROWAVE STUDIO.

been used in a number of experiments [5,7,10], and thus it is chosen here for theoretical consideration. The conditions of low-contrast (small) perturbations of the waveguide wall allow us to apply a modal approximation. We assume that the transverse structure of the excited eigenfield is a superposition of the transverse structures of eigenmodes of the unperturbed cylindrical waveguide partially loaded with a metadielectric. The substitution of the corrugation with a thin metadielectric (Fig. 2) allows us to define the field's complex structure at the lattice interface and include the surface fields in the study. One notes that the surface fields do not exist in a smooth cylindrical waveguide machined from an ideal conductor, and they were ignored in [7]. The electric field will be described as a superposition of volume (subscript $v$ ) and surface (subscript s) fields:

$$
\vec{E}=\vec{A}_{s}+\vec{B}_{v}
$$

Such a description is different from the one used in [7], which is based on defining the structure's eigenmodes as a superposition of partial volume waves structurally coinciding with the eigenmode of the unperturbed waveguide. In this 


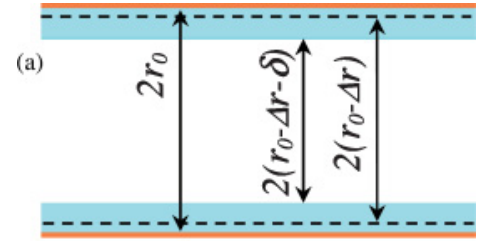

(b)

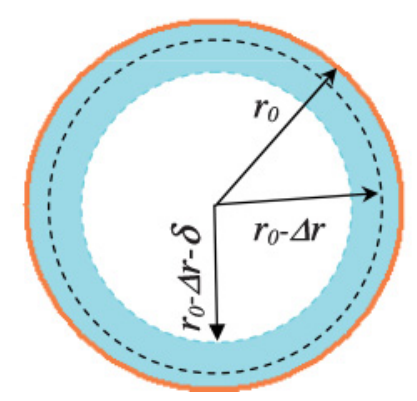

FIG. 2. (Color online) The schematics of the (a) $r-z$ and (b) $r-\varphi$ cross sections of partially loaded cylindrical waveguide.

work, we consider that the surface field is bound to the latticevacuum interface, is therefore localized, and decays towards the structure's axis (i.e., it has an imaginary transverse wave number, while the volume (bulk) field with a real transverse wave number occupies the bulk of the structure. We note that the coupling of otherwise independent fields is mediated by the "soft" boundary (i.e., the 2D lattice). The volume field will be considered as having a transverse structure coinciding with the structure of a near cutoff azimuthally symmetric eigenmode of the unperturbed cylindrical waveguide of radius $r$. The thin dielectric insert does not affect the transverse structure of this partial field, as the near cutoff wave that forms this field has $\lambda_{\mathrm{z}} \sim L$ (where $L$ is the length of the lattice) and $\lambda_{\mathrm{z}} \gg d_{z}$. Taking into account the amplitude of the perturbation $\Delta r$, one finds an approximate expression for the waveguide radius $r=r_{0}-\Delta r$ (Fig. 2). The surface fields (SFs) are described as eigenmodes of the partially loaded cylindrical waveguide. The surface fields will have a real transverse wave number inside the dielectric and an imaginary transverse wave number outside it. The effective dielectric used in the substitution has a refractive index and thickness defined by the lattice parameters, as well as the field's structure and frequency.

Let us consider the stationary regime (i.e., when cavity's eigenmode is established). In this case, the azimuthally nonsymmetric $\left(m_{s} \neq 0\right)$ surface field can be described as a superposition of $E$ and $H$ modes of the partially loaded cylindrical waveguide ( $E H$ hybrid mode). By taking into account the cylindrical topology of the lattice (i.e., periodicities along azimuthal $\varphi$ and longitudinal $z$ coordinates), the Fourier decomposition of the surface field can be applied:

$$
\begin{aligned}
& E_{z}=\sum_{m_{s}} F_{m_{s}}^{e}\left(k_{\perp s} r\right) \sin \left(m_{s} \varphi\right) \sum_{q=-\infty}^{\infty} E_{q}(z) e^{i q \bar{k}_{z} z} ; \\
& H_{z}=\sum_{m_{s}} F_{m_{s}}^{h}\left(k_{\perp s} r\right) \cos \left(m_{s} \varphi\right) \sum_{q=-\infty}^{\infty} H_{q}(z) e^{i q \bar{k}_{z} z},
\end{aligned}
$$

where $E_{z}$ and $H_{z}$ are the longitudinal field components which define the hybrid $E H$ mode, $q$ is the harmonic number due to the lattice periodicity along $z$, and $m_{s}$ is the number of azimuthal variations of the field. The surface field's transverse wave number is $k_{\perp \mathrm{s}}$, the angular frequency is $\omega=k v_{\mathrm{ph}}, k$ is the wave vector, and $v_{\text {ph }}$ is the phase velocity $\left(v_{\mathrm{ph}}=c / n\right.$, where $c$ is speed of light in vacuum and $n$ is the refractive index of the dielectric). The amplitudes $E_{q}(z)$ and $H_{q}(z)$ are the slowly varying amplitudes of the field harmonics, and $F_{m}^{e, h}(x)$ are the cylindrical functions of order $m$. Let us note that outside the metadielectric the functions $F_{m}^{e, h}(x)$ are combinations of modified Bessel functions ( $x$ is imaginary) [8] defining the field decay toward the central axis of the cylindrical structure, while inside the dielectric the field is defined by the ordinary Bessel functions that have an oscillating nature. It is important to note that in general, the boundary of the metadielectric may not coincide with the corrugation boundary (Fig. 2). The surface and volume fields are coupled on the metadielectric-vacuum interface, and the radius of the interface can be found from the impedance matching condition, which is discussed below. The matching condition on the boundary follows also from the two-stage scattering model via surface current excitation (i.e., as the two fields excite the same surface currents, their impedances on the boundary should be equal). The transverse structure of the near cutoff volume field (in the stationary regime) is close to the structure of a $\mathrm{TM}_{0 l}$ mode of cylindrical waveguide and is defined by an ordinary Bessel function $J_{0}(x): E_{z}=J_{0}\left(k_{\perp v} r\right) \sum_{q=-\infty}^{\infty} E_{q}^{v}(z) e^{i q \bar{k}_{z} z}$, where $l$ is the radial variation number and $k_{\perp v}$ is the transverse wave number. To illustrate the applicability of the model, numerical studies of the eigenfields' distributions inside the 2D structure [Fig. 1(b)] have been carried out using the three-dimensional (3D) code MAGIC. The structure studied and shown has square-wave periodic perturbations on the inner surface of the waveguide. The total length of the lattice is $48 \mathrm{~mm}$ and the mean diameter is $79 \mathrm{~mm}$, while the lattice has the following parameters: $\bar{m}=$ 28, $\Delta r=0.5 \mathrm{~mm}$, and $d_{z}=8 \mathrm{~mm}$. To simulate the excitation of the EM field inside the structure, the coaxial launcher tested in the real experiments and based on the coaxial line termination [5] has been modeled [Fig. 1(b)]. In this case, the Transverse Electromagnetic (TEM) wave is formed in a coaxial line before the termination point. In the vicinity of the periodic structure's input, the line is terminated, resulting in excitation of the whole spectrum of the azimuthally symmetric waveguide modes. Figures 3 and 4 illustrate the dependence of the structure eigenfield excited by a narrow band (35-40 GHz) pulse. In Fig. 3, the dependencies of the transverse structures of the electric [Fig. 3(a), bold lines] and magnetic [Fig. 3(b), bold lines] fields on the radial coordinate are shown and compared with the transverse structure of the field associated with the TM near the cutoff wave (broken lines) of the conventional smooth waveguide of the same dimensions. It is evident that the volume fields observed inside the 2D lattice and smooth cylindrical waveguide coincide well and that the differences are due to the surface fields excited at the cavity wall (see $H_{r, z}$ and $E_{\phi}$ field components). The TM near the cutoff, azimuthally symmetric field has zero $H_{r, z}$ and $E_{\phi}$ field components, while the eigenfield structure of the 2D PSL-based cavity has all six nonzero field components, including both longitudinal electric and magnetic fields. This is similar to the hybrid mode of an unperturbed partially dielectric-loaded waveguide, which indirectly confirms the model described above. In spite of 
(a)

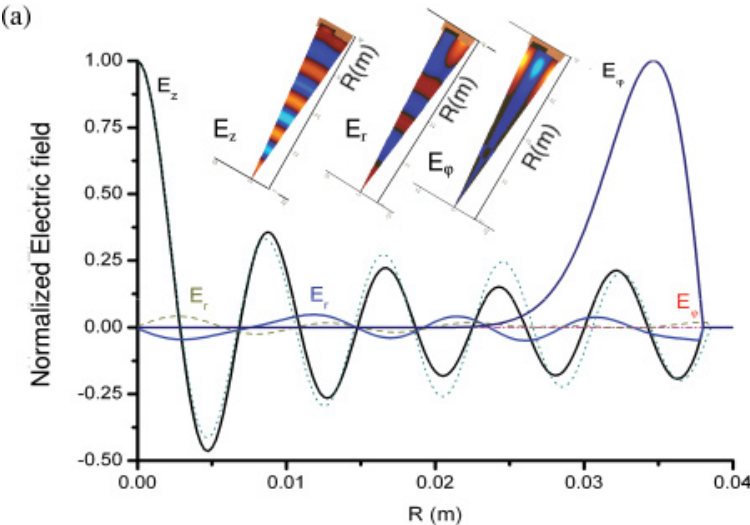

(b)

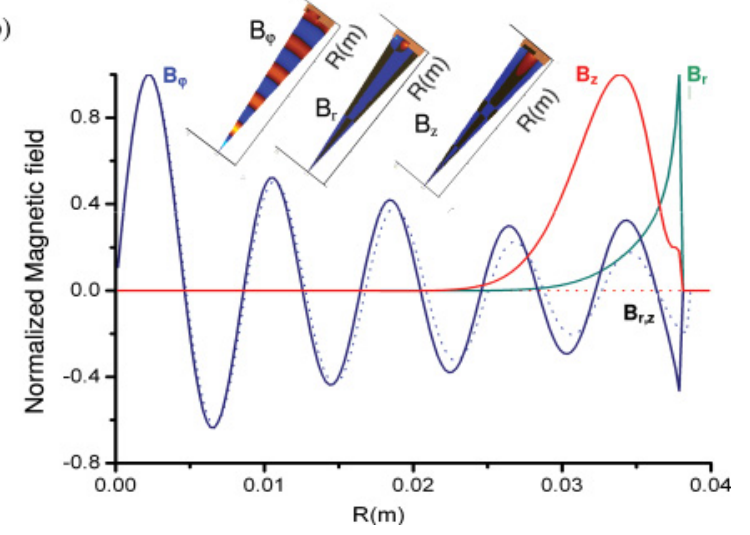

FIG. 3. (Color online) The comparison of the transverse structures of the (a) electric $E_{\mathrm{z}, \mathrm{r}, \phi}$ and (b) magnetic $B_{\mathrm{z}, \mathrm{r}, \phi}$ fields observed (3D code MAGIC) inside the SF cavity (solid lines) and smooth cylindrical waveguide (SCW) cavity (broken lines). The SCW cavity eigenmode's transverse structure coincides with the structure of the near cutoff $\mathrm{TM}_{0,10}$ wave. The inserts (contour diagrams) illustrate (a) electric and (b) magnetic fields inside the SF cavity (the $1 / 28$ th section of the structure).

the fact that the fields $H_{z}$ and $E_{\phi}$ have structures similar to those of a whispering-gallery mode, the $H_{r, \phi}$ components are exponentially decaying toward the center, underlining surface field behavior. The inserts to Figs. 3(a) and 3(b) are the partial contour plots (showing one azimuthal period of the lattice) illustrating the transverse dependence of the periodic structure's eigenmode in the $r-\varphi$ cross sections. In Fig. 4, a full set of contour plots along $r$-z [Fig. 4(a)] and $r-\varphi$ [Fig. 4(b)] are shown. The coupling between the surface and volume fields can be clearly seen. Also, one may note that the decay of the surface fields starts outside the lattice-vacuum interface (the maximum of the field is slightly elevated above the metal surface), allowing us to define the boundary of the metadielectric as the field's caustic radius.

Let us look closely on a field structure excited inside the 2D SF cavity (Fig. 4). We note that each fundamental cell or scatterer supports an individual uncoupled localized field, and the cavity's eigenmode is formed only if such fundamental cells are synchronized [19]. It is known that the resonant coupling of the surface and volume fields on the SP lattice takes place only if the Bragg resonance condition $\overrightarrow{\vec{k}}=\vec{k}_{s}-\vec{k}_{v}$ is satisfied, where $\overrightarrow{\vec{k}}$ is the lattice reciprocal vector and $\vec{k}_{s, v}$ are the wave vectors. The number of the field's azimuthal variations, as well as the longitudinal wave numbers, are linked with the lattice parameters such that $\bar{m}=m_{s}+m_{v}$ and $\bar{k}_{z}=k_{z s}-k_{z v}$. If the eigenmode is formed, and the volume field is azimuthally symmetric $m_{v}=0$, the SF number of azimuthal variations is $m_{s}=\bar{m}$ and one can write $A_{s}(\varphi) e^{i \varphi_{\bar{m}} \bar{m}}=A_{s}\left(\varphi+\varphi_{\bar{m}}\right)$ where $\varphi_{\bar{m}}=2 \pi / \bar{m}$ (Fig. 4). The lattice also defines the localized SF periodicity along the $z$ coordinate [i.e., $A_{s}(z) e^{i \bar{k}_{z} d_{z}}=$ $\left.A_{s}\left(z+d_{z}\right)\right]$, leading to a strong presence of spatial harmonics in the stationary regime. As a result, taking into account that the volume field is represented by a fundamental harmonic of a near cutoff ( $k_{z v} \cong 0$; see Figs. 3 and 4) wave with $m_{v}$ $=0$ (azimuthally symmetric field, Fig. 4), it is clear that the coupling takes place with the \pm 1 spatial harmonics of the SF $\left|k_{z s}\right|=\left|\bar{k}_{z}\right|$, having $m_{s}=\bar{m}$. At this stage, for clarity, the coupling between the higher harmonics of the volume and surface fields are overlooked; however, all these discussions are still valid for higher harmonics as well. Further, we deal with the cavity fundamental eigenmode, which is defined as a superposition of the azimuthally symmetric, near cutoff, volume partial field and surface partial field having $m_{s}=$ $\bar{m}$ and one radial variation. No doubt, more complex modes can also be observed, for instance if the volume wave is not azimuthally symmetric. Considering the fundamental mode, we can rewrite the expressions (2a):

$$
\begin{gathered}
E_{z}^{s}=F_{\bar{m}}\left(k_{\perp s} r\right) E(z) \sin \left(\bar{k}_{z} z\right) \sin (\bar{m} \varphi) ; \\
H_{z}^{s}=F_{\bar{m}}\left(k_{\perp s} r\right) H(z) \cos \left(\bar{k}_{z} z\right) \cos (\bar{m} \varphi) ; \\
E_{z}^{v}=J_{0}\left(k_{\perp v} r\right) .
\end{gathered}
$$

The surface modes with $m \neq 0$, unlike whispering-gallery modes, are always hybrid (i.e., having both $E_{\mathrm{z}}$ and $H_{\mathrm{z}}$ (see below) field components). Due to coupling to a near cutoff $T M_{0,10}$ mode, which is defined by $E_{\mathrm{z}, \mathrm{r}}$ and $H_{\phi}$ with $\left|E_{r}\right| /\left|E_{z}\right|=k_{z} / k_{\perp v} \approx d_{z} / L$, the coupling between the surface and volume fields takes place via the $H_{\phi}$ field [7], leading to excitation and strong modification of the transverse structures of the surface $H_{\phi}$ and $E_{\mathrm{z}}$ field components. The peculiarity of the eigenmode structures observed is due to a combination of ordinary and modified Bessel functions, which "elevates" the maximum amplitude of the surface fields above the latticevacuum interface, making it look like a whispering-gallery mode. However, if the whispering-gallery mode having $m=$ $\bar{m}=28$ would be excited, the $E_{z}$ and $E_{\phi}$ should be comparable, while the $E_{\phi}$ observed in modeling is nearly 100 times smaller than $E_{z}$. Let us note that "elevation" of the SF above the interface makes it more effective for interaction with the active media located outside the lattice.

To identify the fields bound to the lattice, one can also analyze the partial fields' dispersions at the frequency of the interest. The fundamental expressions for the dispersions (in the cylindrical waveguide partially loaded with a metadielectric) for volume and surface fields are $k^{2}=k_{\perp v}^{2}+k_{z}^{2}$ and $k^{2}=k_{z}^{2}-$ $k_{\perp s}^{2}$, respectively. It is known that under the assumption of the perturbations' amplitudes tending to zero, any complex dispersion relation that describes coupling of the partial fields should split into fundamental dispersions of the fields existing in the unperturbed system. In the case considered, the partial fields' dispersion diagrams should coincide with the dispersions of the unperturbed waveguide eigenmodes; however, due to the 
(a)
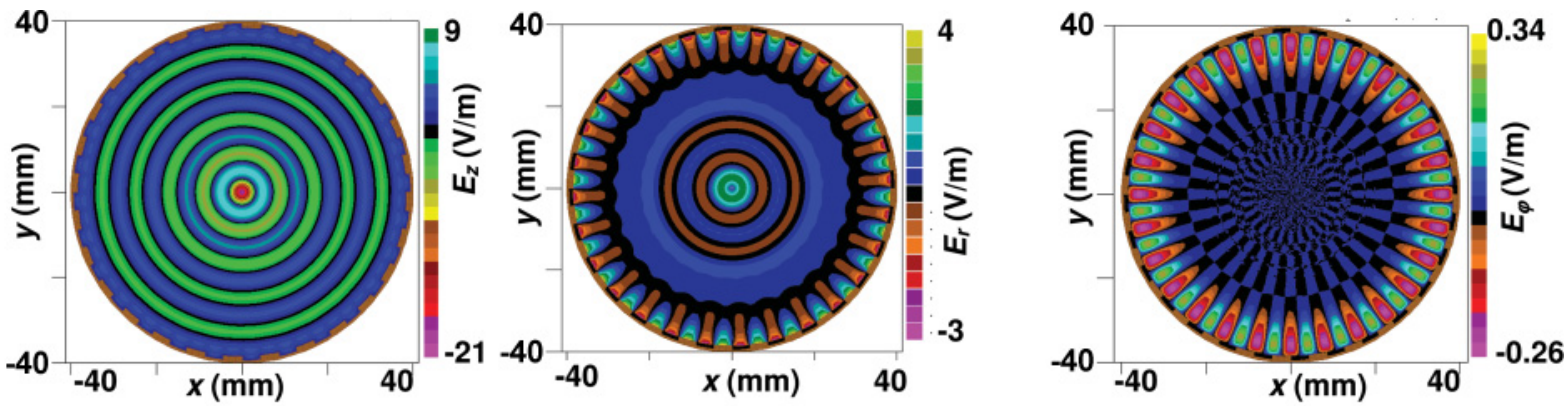

(b)
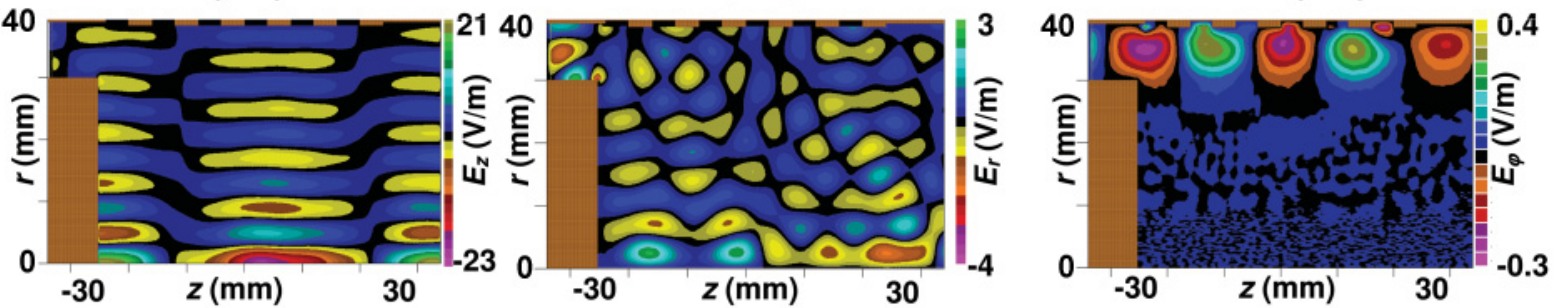

(c)
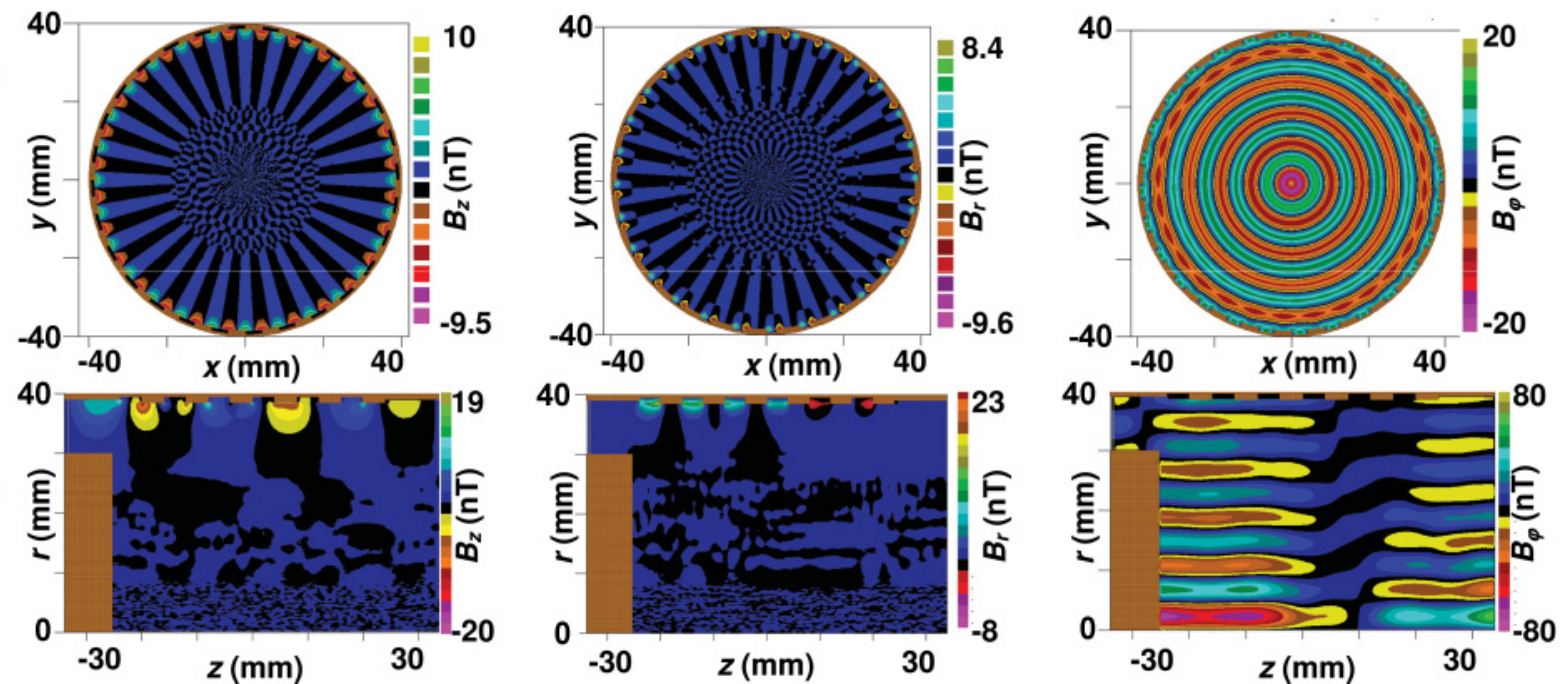

FIG. 4. (Color online) The contour plots of $(\mathrm{a}, \mathrm{b})$ electric $(E)$ field's components and (c,d) magnetic $(B)$ field's components in $(\mathrm{a}, \mathrm{d}) r$ - $z$ and (b,d) $r-\varphi$ cross sections. The contours indicate the field strength and polarity. The figures observed using full 3D code MAGIC when the structure is irradiated by the narrow-band $(35-40 \mathrm{GHz})$, flat-top-spectrum pulse.

periodicity along the longitudinal coordinate, the formation of the spatial harmonics should be taken into account. Though the modifications of the diagrams, such as splitting and kink formation, will take place in the immediate vicinity of the crossings of the dispersions' branches, it will only slightly affect the diagram's overall appearance. Therefore, analyzing the unperturbed dispersions may give a good first inspection and fundamental understanding of the properties and positions of the eigenmodes. Such an analysis can also show which partial fields can be coupled in the frequency region of interest. In Figs. 5, the dispersion diagrams associated with different partial fields are shown. The graphs are for the structure having a 40-mm mean radius, 8-mm longitudinal period, and 28 azimuthal variations. To observe coupling between the partial fields in the vicinity of the cutoff frequency of the $\mathrm{TM}_{0 l}$ mode associated with the volume partial field, it is important that the second partial field has an imaginary transverse wave number. If alternatively the partial fields are those of a whispering-gallery mode, either $\mathrm{TM}_{28,1}$ or $\mathrm{TE}_{28,1}$ having $m_{s} \cong 2 \pi r_{0} / \lambda$ and real transverse wave number, the crossing with the $\mathrm{TM}_{0 l}$ mode takes place at higher frequencies above the region of interest, that is, $f>40 \mathrm{GHz}$ [Fig. 5(a)]. However, if the second partial field is the surface field, it is possible to observe the intersection in the frequency interval between the cutoff frequencies $\sim 37.5$ and $40 \mathrm{GHz}$ [Figs. 5(b)-5(d)]. Let us note that in Figs. $5(\mathrm{a})$ and $5(\mathrm{~d})$ the case $\left(\bar{k}_{z}^{2}=k_{\perp s}^{2}+k_{\perp v}^{2}\right.$ and $\bar{k}_{z}=\sqrt{2} k_{\perp v}$ ) when the branches of the surface and the volume fields' dispersions cross each other at the precise cutoff frequency is also shown. Figures 5(b), 5(c), 5(e), and 5(f) show the dispersions observed for the cases $\bar{k}_{z}^{2}<\left(k_{\perp s}^{2}+k_{\perp v}^{2}\right)$ if $\bar{k}_{z}=k_{\perp v} / 1.1(\mathrm{~b}) ; \bar{k}_{z}=k_{\perp v} / 1.5(\mathrm{c})$; and $\bar{k}_{z}^{2}>\left(k_{\perp s}^{2}+k_{\perp v}^{2}\right)$ if $\bar{k}_{z}=1.5 k_{\perp v}$ (e), $\bar{k}_{z}=2 k_{\perp v}$ (f). The arrows indicate the shifts of the branches of the surface field's dispersion with variation of the lattice period $d_{\mathrm{z}}$. In Figs. 5(b) and 5(c), the branches "move" toward each other, while in Figs. 5(e) and 5(f), the branches "move" outward. If $\bar{k}_{z}^{2}=k_{\perp s}^{2}+k_{\perp v}^{2}$ [Fig. 5(d)], 

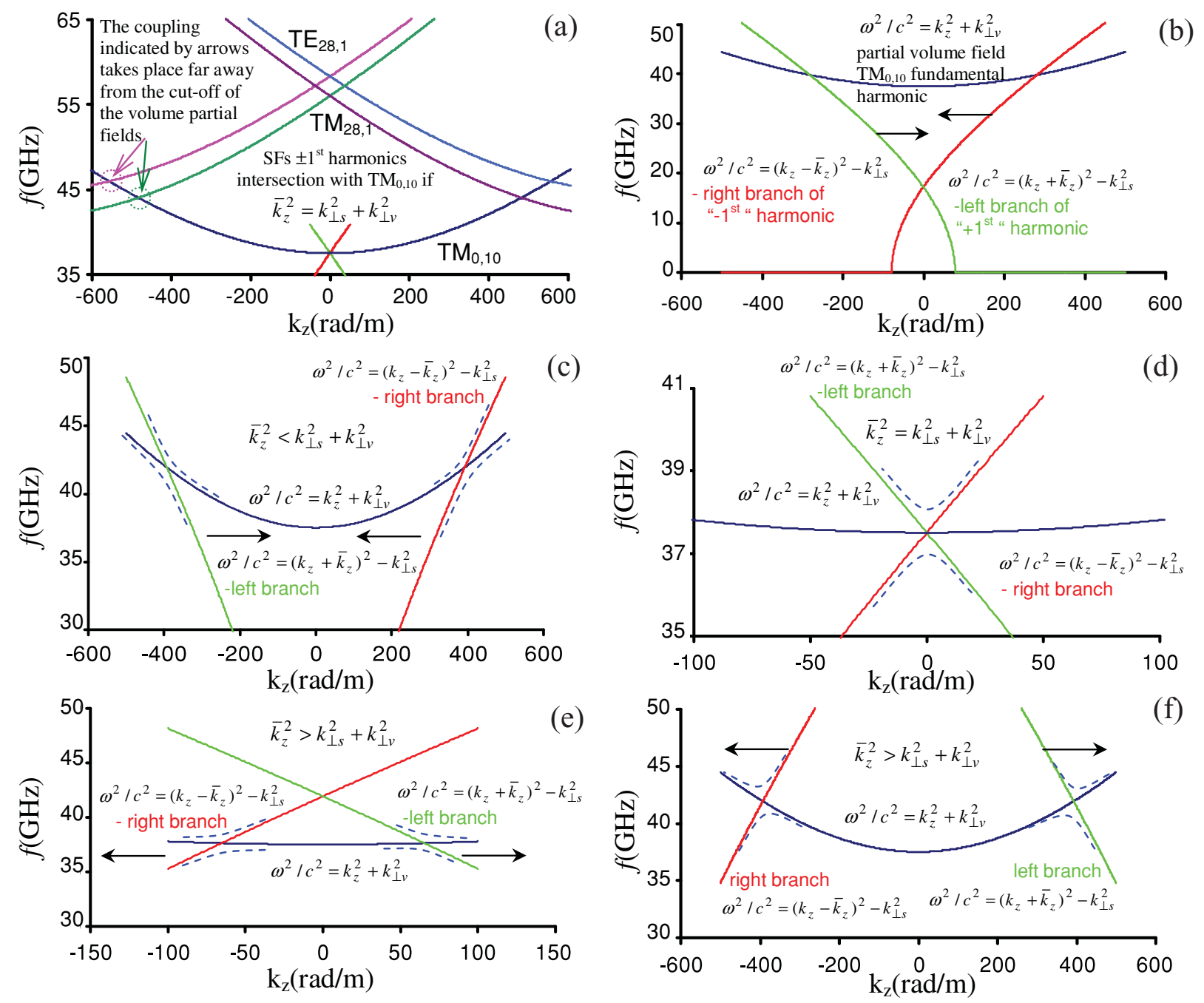

FIG. 5. (Color online) The unperturbed dispersions of the fundamental volume and \pm 1 harmonics surface partial fields observed inside the 2D cylindrical PSL of $80 \mathrm{~mm}$ mean diameter, $\bar{m}=28$, and amplitude of the perturbations tending to zero. The volume field is associated with the $\mathrm{TM}_{0,10}$ mode of the cylindrical waveguide, while the second partial field is associated with (a) whispering-gallery mode $\left(\mathrm{TM}_{28,1}-\mathrm{TE}_{28,1}\right)$ and (b,c,d,e,f) surface field having an imaginary transverse wave number. The crossing between volume and surface field at exact cutoff frequency $\left(\bar{k}_{z}^{2}=k_{\perp s}^{2}+k_{\perp v}^{2}\right.$ ) is shown in (a) and (d). The figures (b) and (c) are observed if $\bar{k}_{z}^{2}<k_{\perp s}^{2}+k_{\perp v}^{2}$, while (e) and (f) if $\bar{k}_{z}^{2}>k_{\perp s}^{2}+k_{\perp v}^{2}$. The arrows indicate the displacement of the surface field dispersions with increase of $\bar{k}_{z}$. The dashed lines indicate schematically the splitting of the dispersions with increase of the lattice contrast.

one observes a bifurcated state. The dispersion splitting is schematically illustrated by dashed lines on these graphs. The topology of the dispersion splittings are different, illustrating the possibilities of observing instabilities of different types, either convective [Figs. 5(b), 5(c)] or absolute [Figs. 5(e), $5(\mathrm{f})]$ instabilities, if the lattice forms an interaction region of an active device driven, for example, by an electron beam. This can be beneficial for devices operating in different regimes and using different types of interactions.

The number of the radial variations of the field excited inside the low-contrast structure is not controlled by the lattice in the same way as it maintains the field's number of azimuthal variations. The number of radial variations only depends on the radius of the unperturbed cylindrical waveguide. Thus, the volume field inside the structure will have $l$ radial variations such that $k_{\perp v}=\chi_{l}^{v} / r$, where $\chi_{l}^{v}$ is the $l$ th root of the Bessel function of zero order. As we deal with the fundamental mode of the structure, inside the frequency interval, which is associated only with the fundamental mode, the number of radial variations of the surface field will be considered to be equal to unity. Increasing the operating frequency will lead to an increase of the surface and volume fields' numbers of radial variations, affecting for instance the inner boundary of the metadielectric (the surface field's radial variations exist only inside the metamedia). The numerical modeling illustrating such eigenfield dependence on the operating frequency and eigenmode excitation has been carried out using the full 3D code MAGIC in the following frequency regions: from 30 to $40 \mathrm{GHz}$ (Fig. 6) and from 65 to $70 \mathrm{GHz}$. Let us note that the first band is well below the whispering-gallery modes' $\left(\mathrm{TE}_{28,1}\right.$ and $\mathrm{TM}_{28,1}$ ) cutoff frequencies $(44.6$ and $41.6 \mathrm{GHz}$ respectively), while the second band was chosen to illustrate 
the concept discussed and to demonstrate the high-order modes' excitation. To observe the cavity eigenmodes having different radial indices, a broad-band pulse has been used. In Fig. 6(a), the eigenmodes excited (solid line) by the pulse with a flat spectrum (dotted line) in the region between 30 and $40 \mathrm{GHz}$ are shown. The spectrum maxima are associated with the eigenmodes having different radial $l \in$ [8-10] and the same azimuthal indices, $m=\bar{m}=28$. An excitation of a specific mode with $l=9$ [Figs. 4 and $6(\mathrm{~b})$, solid line] has been observed using a narrow-band, flat-top-spectrum pulse in the interval from 35 to $40 \mathrm{GHz}$ [Fig. 6(b), dotted line]. The contour plots of the eigenfields' components observed in this case are shown in Fig. 4. In Fig. 7, the contour plots of the eigenfield structure inside the cavity observed as a result of the cavity excitation with a narrow-band, flat-top-spectrum pulse similar to the one shown in Fig. 6(b) (dotted line) in the interval from 65 to $70 \mathrm{GHz}$ are presented. It can be seen from these figures that the surface field's components of high-order eigenmodes are also localized inside a specific region. The number of radial variations is increased, as discussed, while the azimuthal variation number of the eigenmode is maintained constant. The increase of the radial variation number results in a shift of the surface field caustic radius from the lattice toward the center. The dotted lines in Fig. 7 indicate the position of the field caustic radius, which coincides with the metadielectric boundary.
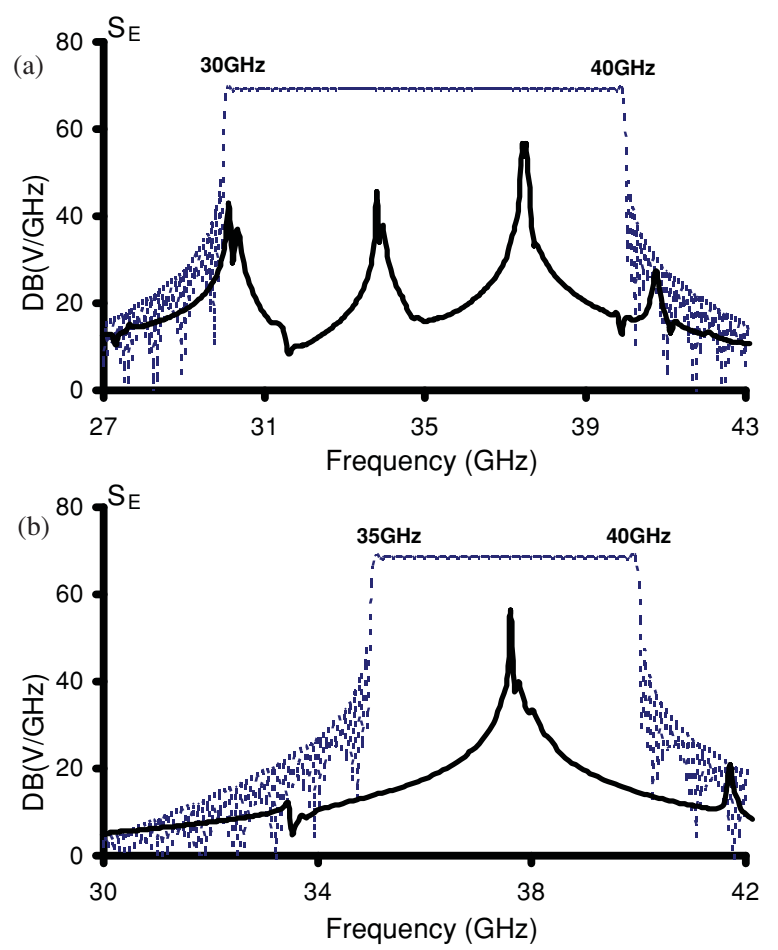

FIG. 6. (Color online) The spectra of the structure's eigenmodes excited (solid lines) if the input signal used to irradiate the lattice has a flat-top spectrum in the frequency ranges (a) $30-40 \mathrm{GHz}$ and (b) 35-40 GHz.

\section{SURFACE FIELD INSIDE WAVEGUIDE PARTIALLY LOADED WITH A METADIELECTRIC}

Let us consider the SF structure observed inside the 2D periodic lattice by substituting the periodic lattice with an effective imaginary dielectric (metadielectric) [18], the thickness $2 \Delta r-\delta$ and refractive index $n=\sqrt{\varepsilon \mu}$ of which are functions of the EM field frequency, structure, and lattice parameters (Fig. 2). The inner and outer radii of the metadielectric insert can be defined as $r_{d}=r_{0}-\Delta r-\delta$ and $r_{+}=r_{0}+\Delta r$ respectively. The surface field is localized (having a real wave number) inside the dielectric $\left(r_{d}<r \leqslant\right.$ $\left.r_{+}\right)$and decaying (imaginary transverse wave number) outside $\left(r \leqslant r_{d}\right)$. At the boundary of the metadielectric, the field's continuity conditions for its tangential components (i.e., $E_{z, \phi}$ and $H_{z, \phi}$ ) should be met. The field is described as a standing (to observe localization) wave having both $E_{z}$ and $H_{z}$ field components and defined using the vector potential $\Psi_{e, h}$ as follows:

$$
\left(E_{z} ; H_{z}\right)=\left(\frac{\partial^{2}}{\partial z^{2}}+k^{2} \varepsilon \mu\right)\left(\Psi_{e} ; \Psi_{h}\right),
$$

where the vector potentials inside the dielectric are

$$
\begin{aligned}
& \Psi_{e}=C_{1} F_{e}\left(\kappa_{s} r\right) \sin (\bar{m} \varphi) \sin (\bar{k} z), \\
& \Psi_{h}=C_{2} F_{h}\left(\kappa_{s} r\right) \cos (\bar{m} \varphi) \cos (\bar{k} z)
\end{aligned}
$$

and those outside the dielectric are

$$
\begin{aligned}
& \Psi_{e}=C_{3} \widehat{F}_{e}\left(p_{s} r\right) \sin (\bar{m} \varphi) \sin (\bar{k} z), \\
& \Psi_{h}=C_{4} \widehat{F}_{h}\left(p_{s} r\right) \cos (\bar{m} \varphi) \cos (\bar{k} z)
\end{aligned}
$$

and

$$
\kappa_{s}^{2}=k^{2} n^{2}-\bar{k}_{z}^{2}, \quad p_{s}^{2}=\bar{k}_{z}^{2}-k^{2} .
$$

The $C_{1,2,3,4}$ are arbitrary constants and

$$
\begin{gathered}
F_{e}\left(\kappa_{s} r\right)=J_{\bar{m}}\left(\kappa_{s} r\right) N_{\bar{m}}\left(\kappa_{s} r_{+}\right)-J_{\bar{m}}\left(\kappa_{s} r_{+}\right) N_{\bar{m}}\left(\kappa_{s} r\right), \\
F_{h}\left(\kappa_{s} r\right)=J_{\bar{m}}\left(\kappa_{s} r\right) N_{\bar{m}}^{\prime}\left(\kappa_{s} r_{+}\right)-J_{\bar{m}}^{\prime}\left(\kappa_{s} r_{+}\right) N_{\bar{m}}\left(\kappa_{s} r\right), \\
\hat{F}_{e}\left(p_{s} r\right)=\widehat{F}_{h}\left(p_{s} r\right)=I_{\bar{m}}\left(p_{s} r\right) .
\end{gathered}
$$

The approach is synonymous with including only the first positive and negative harmonics (observed in the periodic structure), manifesting itself as $\cos (\bar{k} z)$ and $\sin (\bar{k} z)$ dependences of the $\Psi_{e}$ and $\Psi_{h}$ potentials. By expressing the tangential fields using (4) and applying the continuity conditions at the dielectric-vacuum interface, the characteristic equation that links the transverse wave numbers can be observed:

$$
p_{s}^{4} \kappa_{s}^{4} r_{d}^{4}\left(\varepsilon f_{e}-\hat{f}_{e}\right)\left(\mu f_{h}-\hat{f}_{h}\right)=\bar{m}^{2} \bar{k}_{z}^{2} k^{2}\left(n^{2}-1\right)^{2},
$$

where $\hat{f}_{e}=\hat{f}_{h}=\hat{f}=-I_{\bar{m}}^{\prime}(y)\left(y I_{\bar{m}}(y)\right), \quad y=p_{s} r_{d}, \quad f_{e}=$ $\frac{1}{x_{d}} \frac{J_{\bar{m}}^{\prime}\left(x_{d}\right) N_{\bar{m}}\left(x_{+}\right)-J_{\bar{m}}\left(x_{+}\right) N_{\bar{m}}^{\prime}\left(x_{d}\right) N_{\bar{m}}\left(x_{+}\right)-J_{\bar{m}}\left(x_{+}\right) N_{\bar{m}}\left(x_{d}\right)}{J^{\prime}}, \quad f_{h}=\frac{1}{x_{d}} \frac{J_{\bar{m}}^{\prime}\left(x_{d}\right) N_{\bar{m}}^{\prime}\left(x_{+}\right)-J_{\bar{m}}^{\prime}\left(x_{+}\right) N_{\bar{m}}^{\prime}\left(x_{d}\right)}{J_{\bar{m}}\left(x_{d}\right) N_{\bar{m}}^{\prime}\left(x_{+}\right)-J_{\dot{m}}^{\prime}\left(x_{+}\right) N_{\bar{m}}\left(x_{d}\right)}$, and $x_{d,+}=\kappa_{s} r_{d,+}$. The dispersion equation (7) shows that the EM fields with nonzero azimuthal variation numbers are always hybrid (i.e., have $E_{\mathrm{z}}$ and $H_{\mathrm{z}}$ field components), while the azimuthally symmetric fields are either $E$ or $H$ polarized. It also indicates that far from the resonance, where the effective refractive index is equal to 1 (no interaction), no hybrid modes can be observed; that is, the partially loaded 
(a)
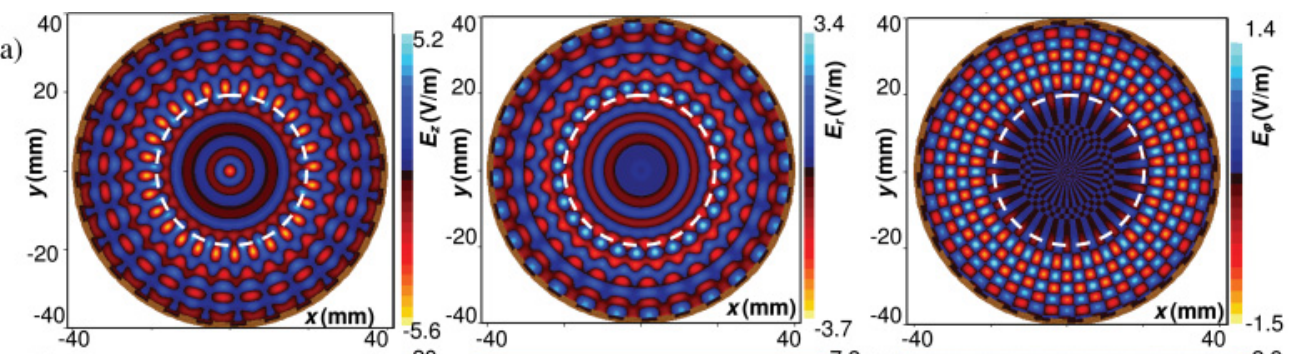

(b)
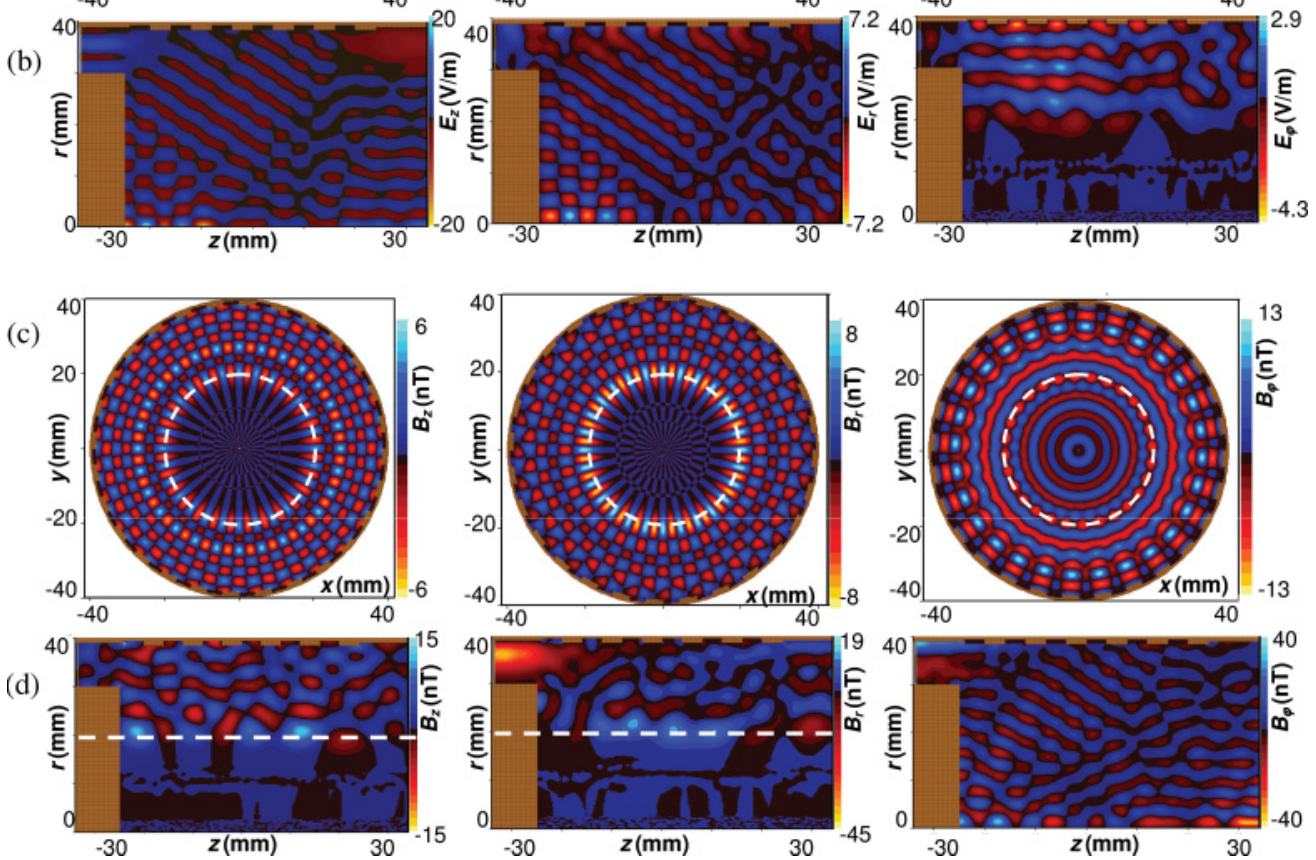

FIG. 7. (Color online) The contour plots of $(\mathrm{a}, \mathrm{b})$ electric $(E)$ field's components and $(\mathrm{c}, \mathrm{d})$ magnetic $(B)$ field's components in $(\mathrm{a}, \mathrm{d}) r$ - $z$ and (b,d) $r-\varphi$ cross sections. The contours indicate the field strength and polarity. The figures were observed using full 3D code MAGIC when the structure was irradiated by the narrow-band $(65-70 \mathrm{GHz})$, flat-top-spectrum pulse. The dotted line shows schematically the boundary of the imaginary metadielectric, whose radius coincides with caustic radius.

waveguide turns into a conventional cylindrical waveguide. Taking into account (5), one finds that

$$
\kappa_{s}^{2}+p_{s}^{2}=k^{2}\left(n^{2}-1\right),
$$

which together with (7) allows the evaluation of the transverse wave numbers and dispersion analysis. To define fully the dispersion relation, the refractive index and dielectric inner boundary have to be found. Assuming that the metadielectric has $\mu=1$, the refractive index $n$, where $n^{2}=\varepsilon \mu$, can be defined from the following consideration: The system which includes the lattice and EM fields (the lattice manifests itself in the appearance of the field's harmonics) has been substituted with a waveguide partially loaded with a metadielectric and the EM field. Thus, taking into account that the localized field, which is nonpropagating and exists only inside the metadielectric, is coupled to the cutoff volume wave, we require the absolute value of $\kappa_{\mathrm{S}}$ (the transverse wave number inside the metadielectric) to be equal to the wave number $k$. One can also regard this as phase matching of the fundamental harmonics of the surface and volume fields. Taking (5) into account leads to the following expression: $k^{2} n^{2}=\kappa_{s}^{2}+\left(l \bar{k}_{z}\right)^{2}=$ $k^{2}+\left(l \bar{k}_{z}\right)^{2}$, where $l$ is an integer indicating the harmonic's number. Considering only the surface field's \pm 1 harmonics, the expression for the refractive index in the vicinity of the resonance frequency takes the following form:

$$
n=\sqrt{1+\frac{\bar{k}_{z}^{2}}{k^{2}}} .
$$

At this stage, the only unknown parameter is the inner radius $r_{d}$ of the metadielectric. We define it from the following consideration. At the caustic boundary, the two partial fields (the surface and volume fields) have to be matched, yielding the following condition:

$$
\begin{gathered}
p_{s} \frac{I_{\bar{m}}\left(p_{s} r_{d}\right)}{I_{\bar{m}}^{\prime}\left(p_{s} r_{d}\right)}=k_{\perp v} \frac{J_{0}\left(k_{\perp v} r_{d}\right)}{J_{0}^{\prime}\left(k_{\perp v} r_{d}\right)} \quad \text { and } \\
\omega / c=\sqrt{\left(k_{\perp v}\right)^{2}+k_{z v}^{2}} \cong k_{\perp v},
\end{gathered}
$$

which defines the inner radius. Its analytical solution can be found under the assumptions that the structure is oversized in comparison with an operating wavelength, the amplitude of the corrugations, and the deviation $\delta\left(r_{d}=r_{-}+\delta\right)$ of the metadielectric boundary from the lattice interface (Fig. 2), which are small in comparison with the operating wavelength 
$\left(\delta / r_{0} \ll 1\right.$ and $\left.\delta / \lambda \ll 1\right)$. The solution for $\delta$ can be found by applying Taylor's expansions to the above expression (10):

$$
\delta \cong \frac{p_{s}^{2}}{\left(k_{\perp v}^{2}-p_{s}^{2}\right)} \frac{r_{-}}{\left(p_{s} r_{-}-\bar{m}\right)} .
$$

The expression (11) allows estimation of the field caustic radius, that is, the boundary at which the surface field starts to decay exponentially. One notes that if $k_{\perp v} \cong k=\omega / c$ and considering only the surface field's \pm 1 harmonics, it follows from (11) and (5) that $\delta=0$ if $\bar{k}_{z}=k=\omega / c$ and $\delta \sim-\varepsilon / k$, where $\varepsilon$ is the detuning $\varepsilon=\left(\bar{k}_{z} / k\right)^{2}-1$. Also, if an interaction between an electron beam and the EM field is considered in order to observe high-power lasing, the electron beam should propagate outside the lattice while still inside the region defined by the shift $\delta$ (i.e., $\delta<0$ ). Thus, expression (11) imposes conditions on the electron beam, accelerating voltage, EM field, and lattice parameters. By optimizing these parameters, a steady-state, single-mode, high-power source operating in the low- $\mathrm{THz}$ frequency range can be created.

\section{CHERENKOV OSCILLATOR BASED ON CYLINDRICAL 2D SURFACE PERIODIC LATTICE}

Let us consider the interaction between an electron beam and the electromagnetic fields mediated by the cylindrical 2D PSL. Inside the cavity defined by the lattice, the partial surface and volume fields form the cavity eigenmode. Electrons interact with the synchronous harmonic of the cavity eigenmode's partial SF at the lattice interface. The near cutoff partial volume field synchronizes the individual scatterers forming the lattice and ensures that the different parts of the oversized electron beam interact with the coherent SF. The condition required for the electron beam-EM wave interaction has the following general form:

$$
\omega=k_{z} v_{z}+\frac{2 \pi}{d_{z}} v_{z}
$$

where $v_{\mathrm{z}}$ is the electron beam longitudinal velocity and $k_{\mathrm{z}}$ is the wave's longitudinal wave number. Taking into account that the electrons interact with the localized surface field $\left(k_{\mathrm{z}}\right.$ $\cong 0)$, (12a) can be rewritten as $f=\frac{c}{d z} \sqrt{1-\gamma^{-2}}$, where $\gamma=$ $1+\frac{e U}{m_{0} c^{2}} \cong 1+\frac{W(\mathrm{keV})}{511 \mathrm{keV}}$ is the relativistic Lorentz factor and $f$ is the wave frequency. By rearranging the last expression, the formula linking the longitudinal period of the lattice with the wave frequency and the electron beam accelerating voltage $U$ can be found:

$$
U(\mathrm{kV}) \cong 511 \mathrm{kV} \times\left[\frac{\lambda}{\sqrt{\lambda^{2}-d_{z}^{2}}}-1\right]
$$

To study the interaction between an electron beam and the cavity eigenmode, it is important to define correctly the radial position of the annular electron beam (i.e., the mean radius). We have to locate the electron beam outside the lattice to avoid its interception with the structure. Such an interception may affect the lifetime of the oscillator as well as limit the level of output power. To optimize the beam radius, the relations (11) and (12b) can be used, and as discussed above, to observe an effective interaction between the electron beam and the EM field the electron beam should be inside the following region (Fig. 2):

$$
r_{0}-\Delta r=r_{+}>R>r_{d}=r_{0}-\Delta r+\delta .
$$

To avoid the electron beam interception, it is important that $\delta<0$. This can be achieved if either

or

$$
k_{\perp v}^{2}-p_{s}^{2}>0, \quad \text { while } \quad p_{s} r_{0}-\bar{m}<0
$$

$$
k_{\perp v}^{2}-p_{s}^{2}<0, \text { while } p_{s} r_{0}-\bar{m}>0 .
$$

By looking at the conditions (13) and linking them to the expression (12b), two distinctive cases can be identified. It is possible to show that the first condition (13a) is associated with high-voltage (relativistic) electron beams, while the condition (13b) is linked to low-voltage (mildly relativistic) electron beams. Indeed, taking into account that $k_{\perp v}=\omega / c, p_{s}=$ $\sqrt{\bar{k}_{z}^{2}-(\omega / c)^{2}}, d_{z}=\frac{\lambda}{a} \sqrt{a^{2}-1}$, and $a^{2}=\left(\frac{U(\mathrm{kV})}{511 \mathrm{kV}}+1\right)^{2}>1$ and substituting them into (11), we find that $k_{\perp v}^{2}-p_{s}^{2}>0$ if $\lambda / d_{z}<\sqrt{2}$, leading to $a>\sqrt{2}$ (i.e., if the beam accelerating voltage is above $U_{0}=212 \mathrm{kV}$ ). In this case, $\delta<0$ if $p_{s} r_{0}-\bar{m}<0$, which is achieved when

$$
\frac{2 \pi r_{0}}{\lambda a}<\bar{m} \quad \text { and } \quad a>\sqrt{2} \text {. }
$$

This shows that for a specific mean radius of the lattice and operating wavelength, the number of the lattice azimuthal variations should be larger than the number of wavelengths along the unperturbed circumference of the waveguide. In the low-voltage regime for which $k_{\perp v}^{2}-p_{s}^{2}<0$ and the electron beam accelerating voltage is less than $U_{0}$, then $\delta<0$ if

$$
\frac{2 \pi r_{0}}{\lambda a}>\bar{m} \text { and } a \sim 1,
$$

indicating that the number of azimuthal variations should be less than the number of wavelengths along the unperturbed circumference of the waveguide. If the conditions (14) are not satisfied, the coupling between the electron beam and electromagnetic fields will be significantly weaker, resulting in the necessity to either increase the length of the interaction region or the electron beam current and propagate the electron beam inside the periodic structure. The electron beam accelerating voltage defines also the topology of the lattice's single cell. If $\gamma$ is large (relativistic electron beam), the cell has rectangular geometry, with the longest side codirected with the electron beam drift velocity, while if $\gamma$ is low (mildly relativistic electron beam), the cells have elongated geometry with the longest side perpendicular to the electron beam drift velocity. One also notes that there is overlap $\Delta$ $\sim(1-1 / a)$ between (14a) and (14b), allowing the maser's operating frequency to be maintained if the accelerating voltage is varied by adjusting only the structure's longitudinal period.

Numerical studies of a 200-GHz Cherenkov oscillator based on the PSL and driven by an annular $\left(r_{\mathrm{b}}=5 \mathrm{~mm}\right)$, thin-wall $(\delta r=0.5 \mathrm{~mm}$ ) electron beam immersed in a guiding magnetic field of $2 \mathrm{~T}$ have been carried out using the $3 \mathrm{D}$ code MAGIC. In Fig. 8, the results illustrating the scaling relation (12b) are shown. The graphs presented were observed under the condition that the system's only variable parameters are 
the electron beam accelerating voltage and the longitudinal period of the structure. The rest of the parameters, including the number of lattice periods along $z$, have been maintained constant. The oscillator's parameters were chosen to satisfy the relations (11) and (14) for the range of the electron beam voltages (overlapping region is from 100 to $300 \mathrm{kV}$ ). It can be seen from Fig. 8 that by changing the electron beam accelerating voltage and scaling the lattice period $d_{\mathrm{z}}$ using (12b), the operating frequency is maintained constant. However, as one would expect, the operating regime as well as the output efficiency of the oscillator varies with the change of the accelerating voltage. In Fig. 8(b), the steady-state singlemode operation of a $0.2-\mathrm{THz}$ Cherenkov oscillator is observed. The device is driven by a $250-\mathrm{kV}, 20-\mathrm{A}$ annular electron beam and the efficiency observed is around $2.5 \%$, yielding $\sim 120 \mathrm{~kW}$ output power. However, taking into account the possibility of highly efficient electron beam energy recovery, which is routinely carried out, an overall efficiency above $40 \%$ (after energy recovery) can be expected. By optimizing the interaction region parameters, a further increase of the energy extraction efficiency and thus an increase of output power can be observed. For instance, an efficiency of $10 \%$ is achieved when a $100-\mathrm{kV}$ electron beam is used [Fig. 8(c)]. However, for the parameters used, output power modulation can be seen, indicating mode competition due to the excitation of high-order eigenmodes. The number of the lattice azimuthal variation $\bar{m}$ should also affect the behavior of the Cherenkov oscillator [see (14)]. The numerical modeling of the Cherenkov oscillator driven by a low-voltage $(50-\mathrm{kV}), 40$-A electron beam was carried out, and the results of the investigation of the dependence of the oscillator operation on $\bar{m}$ are shown in Fig. 9. In these studies, the lattice longitudinal period $d_{\mathrm{z}}=$ $0.58 \mathrm{~mm}$ was changed in accordance with $(12 \mathrm{~b})$ to maintain the operating frequency range of $\sim 0.2 \mathrm{THz}$. The rest of the lattice parameters are the same as listed in Fig. 8. One notes that if the number of the structure's azimuthal variations are maintained ( $\bar{m}=20$ as in Fig. 8 ), a drop of the output power [Fig. 9(a)] and a frequency jump to $207.3 \mathrm{GHz}$ [Fig. 9(b)] [which follows from (14b) take place (operating with higher order radial mode). Taking into account that $r_{0}=5.5 \mathrm{~mm}$, $U=50 \mathrm{kV}(a \sim 1.1), \lambda=1.53 \mathrm{~mm}(\sim 195 \mathrm{GHz})$, one finds that in accordance with condition (14b) $\bar{m}<20$ is required to observe an effective interaction below $195 \mathrm{GHz}$. The variation of transient times observed [Fig. 9(a)] from approximately $4 \mathrm{~ns}$ (for $\bar{m}=18$ ) to above $10 \mathrm{~ns}$ (for $\bar{m}=21$ ) indicates also the dependence of coupling between the electron beam and the EM field on the azimuthal index $\bar{m}$. It is clear that changing $\bar{m}$ results in variation of the oscillation evolution [Fig. 9(a)] and the operating frequency [Fig. 9(b)]. One notes that in all cases observed (14b) has been satisfied. Thus with the change of the number of azimuthal variations, the operating frequency upshift from $182 \mathrm{GHz}(\bar{m}=18)$ to $214 \mathrm{GHz}$ $(\bar{m}=21)$ has been observed. Further increase of $\bar{m}$ led to continuous increase of the transient time, and at $\bar{m}=24$ the startup of the oscillations did not take place. We note that for $\bar{m}=21$ the transition time to observe steady-state single-mode operation has increased from $\sim 7 \mathrm{~ns}$ (for $\bar{m} \in[18 ; 20]$ ) to 30 ns. However, one also notes that the output power has (a)

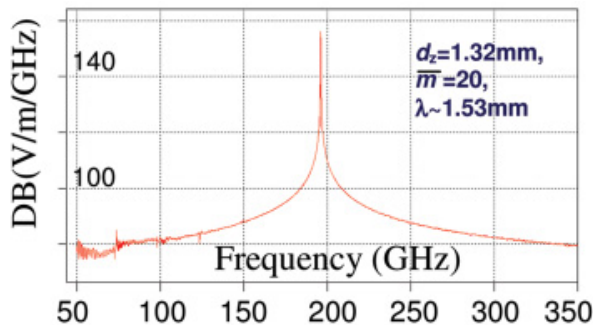

(b)

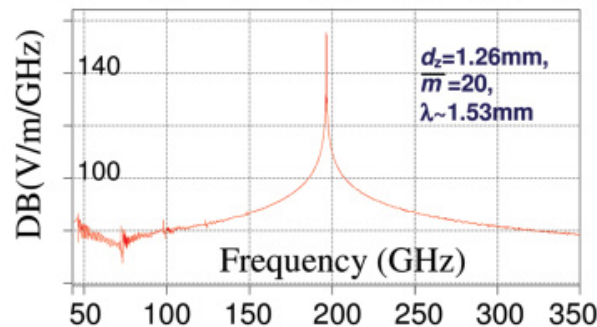

(c)

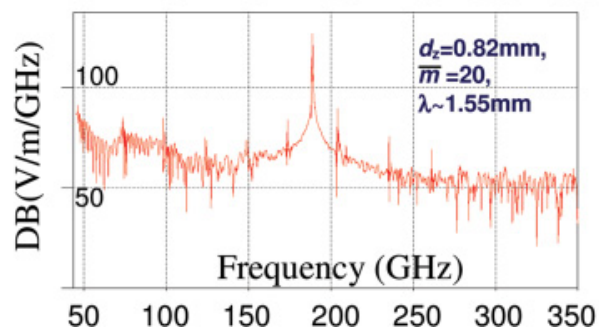

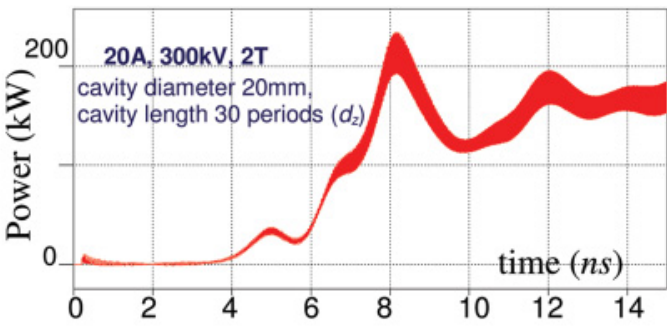
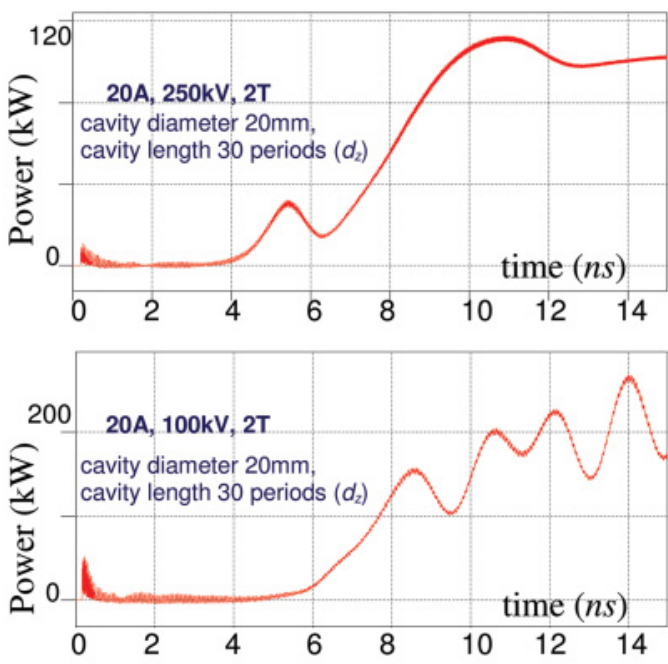

FIG. 8. (Color online) The spectrum of the output radiation (first column) and output power (second column) from Cherenkov oscillator driven by a 20-A, thin, annular electron beam with interaction region formed by cylindrical 2D PSL of $11 \mathrm{~mm}$ diameter with $\bar{m}=20$ and 30 longitudinal periods. The lattice's longitudinal period and beam accelerating voltage was (a) $d_{\mathrm{z}}=1.32 \mathrm{~mm}, U=300 \mathrm{kV}$; (b) $d_{\mathrm{z}}=1.26 \mathrm{~mm}$, $U=250 \mathrm{kV}$; and (c) $d_{\mathrm{z}}=0.82 \mathrm{~mm}, U=100 \mathrm{kV}$. 
(a)

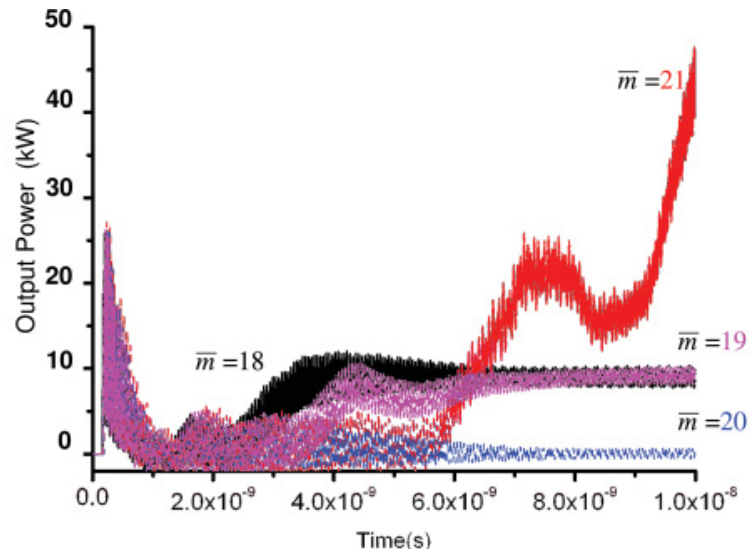

(b)

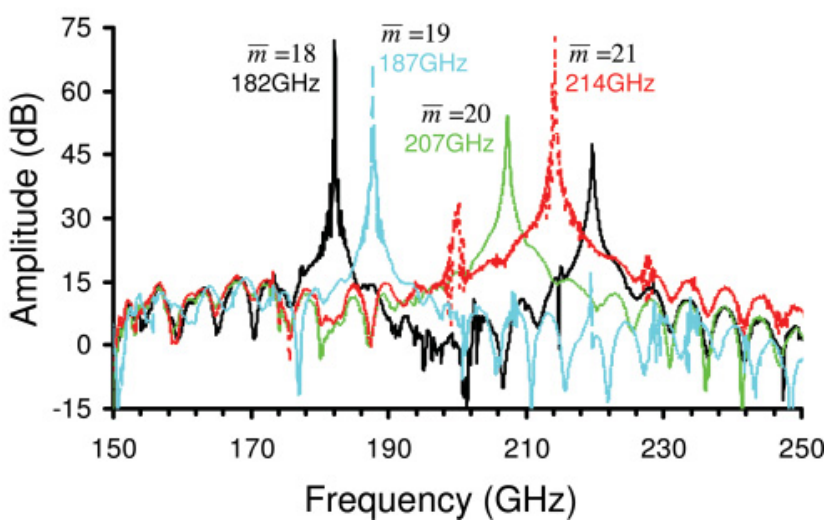

FIG. 9. (Color online) (a) The output power and (b) the spectrum of the output radiation observed from the Cherenkov maser driven by a thin, annular, 40-A, 50-kV electron beam and based on a 2D PSL of $11 \mathrm{~mm}$ mean diameter and $17.4 \mathrm{~mm}$ length (30 longitudinal periods) and having $\bar{m}=[18 ; 21]$.

also increased by a factor of 4 , indicating significant potential for further improvement of the oscillator performance. The excitation of up- and down-shifted satellite modes associated with fields having different radial variation numbers has been observed for $\bar{m} \in[18 ; 21]$. The field amplitudes of these modes are $30 \mathrm{~dB}$ less than the operating mode but still would be measurable if experimental studies are conducted with such a device.

\section{CONCLUSION}

In this paper, we presented the model describing the fundamental eigenmode structure inside the cavity formed by a $2 \mathrm{D}$ periodic surface lattice of cylindrical topology. We demonstrated that the eigenmode structure can be considered as a superposition of partial surface and volume fields, which are coupled on the lattice-vacuum interface. We discussed the structures of the volume and surface partial fields, and it was shown that the surface field excited is different from a whispering-gallery mode and has all the features of a surface mode. To define the structure of the eigenfield, the cylindrical lattice was substituted with a smooth cylindrical waveguide, partially loaded with a metadielectric. It was found that the properties of the metadielectric depend on the lattice and radiation parameters, and the conditions required to observe the elevation of the surface field above the lattice were discussed. Contour plots of the cavity eigenfield structures were demonstrated. By analyzing the dispersion of the weakly coupled partial fields, we illustrated that to observe coupling at a near cutoff frequency of the volume field, the surface field has to decay toward the center (i.e., it should have an imaginary transverse wave number), thus making its structure different from the whispering-gallery modes. Using the observed results, numerical studies of a Cherenkov oscillator based on a 2D PSL cavity and driven by an annular, electron beam were carried out. The dependence of the lattice parameters on the electron beam accelerating voltage was discussed and the required lattice parameters' scaling (to maintain the operation of the Cherenkov oscillator) with variation of the electron beam voltage was shown. Using the 3D numerical code MAGIC, we demonstrated that single-mode steady-state operation of a high-power 200-GHz Cherenkov maser can be achieved.

Let us note that the results observed in the paper have a fundamental impact as they illustrate coupling on a "soft" boundary of two different electromagnetic fields, which without the lattice would be uncoupled. The model developed shows that the set of discrete synchronized scatterers (oscillators) assembled into the 2D cylindrical lattice allows coupling between an azimuthally nonsymmetric, localized surface field and an azimuthally symmetric volume field. The nature of the scatterers as well as the fields coupled can be different from the ones considered, as the mathematical apparatus and concepts used here can be applied to different systems. For instance, similar results showing the evolution of Bose-Einstein condensate density waves in atomic quantum corrals have been recently observed in [21]. Such coupling can also be expected inside the structures formed by dust particles in plasma and other cylindrical systems based on periodic discrete oscillators or scatterers. We also presented the fundamental concept of substituting the PSL with a continuous metadielectric and have shown the possibility of using surface waves for high-power sources of coherent radiation.
[1] N. E. Glass, Phys. Rev. A 36, 5235 (1987); L. Schächter and A. Ron, ibid. 40, 876 (1989); S. E. Korbly, A. S. Kesar, J. R. Sirigiri, and R. J. Temkin, Phys. Rev. Lett. 94, 054803 (2005); B.-I. Wu, J. Lu, J. A. Kong, and M. Chen, J. Appl. Phys. 102, 114907 (2007).

[2] A. Halperin, A. Gover, and A. Yariv, Phys. Rev. A 50, 3316 (1994); J. Urata, M. Goldshtein, M. F. Kimmitt, A. Naumov, C. Platt, and J. E. Walsh, Phys. Rev. Lett. 80, 516 (1998).
[3] A. G. York, H. M. Milchberg, J. P. Palastro, and T. M. Antonsen, Phys. Rev. Lett. 100, 195001 (2008); A. Mizrahi and L. Schächter, Phys. Rev. E 74, 036504 (2006).

[4] M. A. Shapiro, K. R. Samokhvalova, J. R. Sirigiri, R. J. Temkin, and G. Shvets, J. Appl. Phys. 104, 103107 (2008); E. I. Smirnova, A. S. Kesar, I. Mastovsky, M. A. Shapiro, and R. J. Temkin, Phys. Rev. Lett. 95, 074801 (2005). 
[5] I. V. Konoplev, L. Fisher, K. Ronald, A. W. Cross, A. D. R. Phelps, and M. Thumm, Appl. Phys. Lett. 97, 261102 (2010).

[6] V. L. Bratman, B. S. Dumesh, A. E. Fedotov, P. B. Makhalov, B. Z. Movshevich, and F. S. Rusin, IEEE Trans. Plasma Sci. 38, 1466 (2010); Yu. A. Grishin, M. R. Fuchs, A. Schnegg, A. A. Dubinskii, B. S. Dumesh, F. S. Rusin, V. L. Bratman, and K. Möbius, Rev. Sci. Instrum. 75, 2926 (2004); V. L. Bratman, G. G. Denisov, M. M. Ofitserov, S. D. Korovin, S. D. Polevin, and V. V. Rostov, IEEE Trans. Plasma Sci. 15, 2 (1987).

[7] N. S. Ginzburg, I. V. Konoplev, and A. S. Sergeev, Tech. Phys. 41, 465 (1996); I. V. Konoplev, A. W. Cross, W. He, A. D. R. Phelps, K. Ronald, G. R. M. Robb, C. G. Whyte, N. S. Ginzburg, N. Yu. Peskov, and A. S. Sergeev, Nucl. Instrum. Methods Phys. Res. Sect. A 445, 236 (2000); A. W. Cross, I. V. Konoplev, A. D. R. Phelps, and K. Ronald, J. Appl. Phys. 93, 2208 (2003); I. V. Konoplev, A. W. Cross, P. MacInnes, W. He, C. G. Whyte, A. D. R. Phelps, C. W. Robertson, K. Ronald, and A. R. Young, Appl. Phys. Lett. 89, 171503 (2006); I. V. Konoplev, A. W. Cross, A. D. R. Phelps, W. He, K. Ronald, C. G. Whyte, C. W. Robertson, P. MacInnes, N. S. Ginzburg, N. Y. Peskov, A. S. Sergeev, V. Y. Zaslavsky, and M. Thumm, Phys. Rev. E 76, 056406 (2007); I. V. Konoplev, A. W. Cross, P. MacInnes, W. He, A. D. R. Phelps, C. G. Whyte, K. Ronald, and C. W. Robertson, Appl. Phys. Lett. 92, 211501 (2008).

[8] A. M. Cook, R. Tikhoplav, S. Y. Tochitsky, G. Travish, O. B. Williams, and J. B. Rosenzweig, Phys. Rev. Lett. 103, 095003 (2009); G. Adamo, K. F. MacDonald, Y. H. Fu, C.-M. Wang, D. P. Tsai, F. J. Garcia de Abajo, and N. I. Zheludev, ibid. 103, 113901 (2009).

[9] J. C. Ashley, T. L. Ferrell, and R. H. Ritchie, Phys. Rev. B 10, 554 (1974); V. Karagodsky, D. Schieber, and L. Schächter, Phys. Rev. Lett. 104, 024801 (2010).

[10] G. Burt, S. V. Samsonov, K. Ronald, G. G. Denisov, A. R. Young, V. L. Bratman, A. D. R. Phelps, A. W. Cross, I. V. Konoplev, W. He, J. Thomson, and C. G. Whyte, Phys. Rev. E 70, 046402 (2004); I. V. Konoplev, A. D. R. Phelps, A. W. Cross, and K. Ronald, ibid. 68, 066613 (2003); I. V. Konoplev, P. MacInnes, A. W. Cross, A. D. R. Phelps, L. Fisher, and K. Ronald, Appl. Phys. Lett. 93, 231110 (2008).

[11] J. B. Pendry, L. Martin-Moreno, and F. J. Garcia-Vidal, Science 305, 847 (2004); K. Ishizaki and S. Noda, Nature (London) 460,
367 (2009); P. T. Worthing and W. L. Barnes, Appl. Phys. Lett. 79, 3035 (2001).

[12] J. B. Pendry, A. J. Holden, W. J. Stewart, and I. Youngs, Phys. Rev. Lett. 76, 4773 (1996); M. C. K. Wiltshire, J. B. Pendry, I. R. Young, D. J. Larkman, D. J. Gilberdale, and J. V. Hajnal, Science 291, 849 (2001).

[13] R. A. Shelby, D. R. Smith, and S. Schultz, Science 292, 77 (2001); Y. A. Urzhumov and D. R. Smith, Phys. Rev. Lett. 105, 163901 (2010).

[14] D. J. Bergman and M. I. Stockman, Phys. Rev. Lett. 90, 027402 (2003); M. I. Stockman, Nature Photonics 2, 327 (2008); C. Luo, M. Ibanesco, S. G. Johnson, and J. D. Joannopoulos, Science 299, 368 (2003); M. A. Noginov, G. Zhu, A. M. Belgrave, R. Bakker, V. M. Shalaev, E. E. Narimanov, S. Stout, E. Herz, T. Suteewong, and U. Wiesner, Nature (London) 460, 1110 (2009).

[15] N. Aközbek and S. John, Phys. Rev. E 57, 2287 (1998); P. Colman, C. Husko, S. Combre, I. Sagnes, C. W. Wong, and A. De Rossi, Nature Photonics 4, 862 (2010); S. A. Maier, S. R. Andrews, L. Martin-Moreno, and F. J. Garcia-Vidal, Phys. Rev. Lett. 97, 176805 (2006).

[16] F. J. Garcia-Vidal and J. B. Pendry, Phys. Rev. Lett. 77, 1163 (1996); F. Benabid, J. C. Knight, G. Antonopoulos, P. St. J. Russell, Science 298, 399 (2002); J. T. Hugall, J. J. Baumberg, and S. Mahajan, Appl. Phys. Lett. 95, 141111 (2009).

[17] A. Babuty, A. Bousseksou, J.-P. Tetienne, I. Moldovan Doyen, C. Sirtori, G. Beaudoin, I. Sagnes, Y. De Wilde, and R. Colombelli, Phys. Rev. Lett. 104, 226806 (2010); T. Dufaux, J. Dorfmuller, R. Vogelgesang, M. Burghard, and K. Kern, Appl. Phys. Lett. 97, 161110 (2010); A. Schneider. A. Shuvaev, S. Engelbrecht, S. O. Demokritov, and A. Pimenov, Phys. Rev. Lett. 103, 103907 (2009).

[18] E. Lier, D. H. Werner, C. P. Scarborough, Q. Wu, and J. A. Bossard, Nat. Mater. 2950 (2011).

[19] V. A. Fedotov, N. Papasinakis, E. Plum, A. Bitzer, M. Walther, P. Kuo, D. P. Tsai, and N. I. Zheludev, Phys. Rev. Lett. 104, 223901 (2010); V. G. Kravets, F. Schedin, and A. N. Grigorenko, Phys. Rev. Lett. 101, 087403 (2008); R. Singh, C. Rockstuhl, and W. Zhang, Appl. Phys. Lett. 97, 241108 (2010).

[20] J. D. Jackson, Classical Electrodynamics, 3rd ed. (John Wiley \& Sons, New York (1999); A. V. Zayats, I. I. Smolyaninov, and A. A. Maradudin, Phys. Rep. 408, 131 (2005).

[21] H. Xiong and B. Wu, Phys. Rev. A 82, 053634 (2010). 